\title{
Seleção dos parâmetros através dos custos de soldagem para aplicação de revestimento com ligas de níquel depositadas pelo processo MIG/MAG
}

\section{(Using the welding costs to select welding parameters for Weld Overlay with nickel alloy through the GMAW process)}

\author{
Antonio Rodolfo Paulino Pessoa ${ }^{1}$, Hélio Cordeiro de Miranda ${ }^{1}$, Jesualdo Pereira Farias ${ }^{1}$, Willys Machado Aguiar ${ }^{2}$, Francisco de Assis \\ Filho ${ }^{3}$. \\ ${ }^{1}$ Universidade Federal do Ceará, Departamento de Engenharia Metalúrgica e de Materiais, Fortaleza, Ceará, Brasil, antrodolfo. \\ pessoa@bol.com.br \\ ${ }^{2}$ Instituto Federal de Educação, Ciência e Tecnologia do Ceará, Departamento de Indústria, Fortaleza, Ceará, Brasil \\ ${ }^{3}$ Universidade Federal do Ceará, Departamento de Engenharia Mecânica e de Produção, Fortaleza, Ceará, Brasil
}

\begin{abstract}
Resumo
Neste trabalho foram calculados e comparados os custos de soldagem associados a cada um dos ensaios realizados, como forma de determinar parâmetros operacionais adequados e economicamente viáveis para soldagem com o processo MIG/MAG com transferência metálica por curto-circuito, visando uma correta deposição de revestimentos com ligas de níquel sobre um substrato de aço C-Mn. $O$ número de ensaios a serem realizados, foi determinado pelo uso do método Taguchi que dividiu os ensaios em duas matrizes L9, resultando num total de 18 ensaios. Na comparação dos gastos despendidos em cada um dos ensaios foi utilizado o custo direto total da soldagem de um revestimento com $55 \mathrm{~mm}$ de largura e $190 \mathrm{~mm}$ de comprimento, utilizando uma sobreposição de $50 \%$. Com base nos resultados dos ensaios foram pré-selecionados quatro ensaios $(R 7, R 11, R 12$ e RC4), dentre os quais o ensaio R11 apresentou o menor custo. Mas vale ressaltar que nos quatro ensaios pré-selecionados o material de adição utilizado foi a liga 625, pois esta liga apresentar um custo do arame-eletrodo muito inferior as demais ligas (C-276 e 686). Assim a escolha do material de adição para aplicação do revestimento ficou restrita somente a liga 625.
\end{abstract}

Palavras-chave: Custos de soldagem; Revestimento; Ligas de níquel, MIG/MAG e Curto-Circuito.

Abstract: In this work were calculated and compared the welding costs for each of the tests performed to determine operational parameters and economically feasible for welding with GMAW process in a short circuiting transfer mode, aiming at a correct weld overlay with nickel alloy on a substrate of C-Mn steel. The number of tests to be performed was determined by using the Taguchi method that divided the tests into two L9 orthogonal arrays resulting in a total of 18 experiments. To compare the costs incurred in each of the tests we used the total direct cost of welding a layer with dimensions of $55 \times 190 \mathrm{~mm}$, using an overlap of $50 \%$. Based on the results of the tests were pre-selected four tests (R7, R11, R12 and RC4), among which the test R11 has the lowest cost. But it is noteworthy that the four pre-selected test alloy 625 was used as filler metal, because the electrode wire of this alloy has a much lower cost compared to other alloys (C-276 and 686). Thus the selection of the filler metal for weld overlay was restricted only to alloy 625.

Key-words: Welding Costs; Weld Overlay; Nickel alloys; GMAW and Short circuiting.

\section{Introdução}

Como forma de solucionar os problemas relativos à vida útil dos equipamentos e reduzir o número de paradas não programadas (inesperadas) para realização de reparos, a PETROBRAS através de estudos preliminares do Centro de Pesquisa da Petrobrás - CENPES indicam o uso das ligas de níquel como uma das alternativas para adequar ou substituir componentes metálicos que compõem os equipamentos das unidades de produção e refino. Apesar das ligas de níquel constituírem uma alternativa viável para substituir ou adequar os equipamentos empregados nestas

(Recebido em 01/02/2011; Texto final em 31/03/2011).

Artigo originalmente publicado no CONSOLDA2010 unidades, o elevado custo de fabricação de equipamentos ou de componentes maciços constituídos por estas ligas fazem com que os mesmos sejam normalmente construídos com materiais menos nobres, como aços C-Mn e baixa liga e posteriormente revestidos com materiais de excelente resistência à corrosão.

A aplicação de revestimentos em equipamentos industriais pode ser feita na forma de "clad", "lining" e "overlay". Esta última é realizada através da deposição de uma camada de material com características desejáveis (resistência à corrosão, resistência ao desgaste e etc) sobre um substrato. Dentre os vários processos de soldagem utilizados para a aplicação de revestimentos destacam-se: MIG/MAG, arame tubular, arco submerso o TIG com alimentação de arame (frio e quente) e o plasma por arco transferido (PTA).

Mesmo as operações de revestimentos com ligas de níquel ainda possuem custos relativamente elevados e como na maioria 
das vezes, o custo final de um produto ou serviço é um fator determinante para se tomar decisões no âmbito da engenharia.

Assim o objetivo deste trabalho é calcular e comparar os custos de soldagem associados a cada um dos ensaios realizados e com isso determinar parâmetros operacionais adequados e economicamente viáveis para soldagem com o processo MIG/ MAG operando em modo de tensão constante e transferência metálica por curto-circuito, visando uma correta deposição de revestimentos com ligas de níquel sobre um substrato de aço C-Mn.

\section{Matérias e Métodos}

Aseguir serão descritos todos os procedimentos experimentais empregados nos ensaios, bem como os equipamentos, acessórios e os materiais de consumo utilizados.

\subsection{Materiais de consumo}

Os materiais de consumo foram agrupados em três categorias: os corpos de prova, os arames eletrodo e os gases de proteção.

\subsubsection{Corpos de prova}

O material dos corpos de prova utilizados nos ensaios foram placas de aço ASTM A516 grau 60 com dimensões de 200 x 50 $\mathrm{x} 12,7 \mathrm{~mm}$.

\subsubsection{Arame eletrodo}

Foram utilizados como metais de adição os seguintes arames eletrodo ( $\varnothing=1,2 \mathrm{~mm}$ ): o AWS ERNiCrMo-3 (UNSN06625), o AWS ERNiCrMo-4 (UNSN10276) e o AWS ERNiCrMo-14 (UNSN06686).

\subsubsection{Gás de Proteção}

Nos ensaios foram utilizados três gases de proteção: Argônio Puro (100\%Ar), uma mistura gasosa contendo $70 \%$ de argônio e $30 \%$ de Hélio $(70 \% \mathrm{Ar}+30 \% \mathrm{He})$ e uma mistura gasosa contendo $99,97 \%$ de argônio e $0,03 \%$ de óxido nítrico $(99,97 \% \mathrm{Ar}+$ $0,03 \% \mathrm{NO})$.

\subsection{Equipamentos e acessórios}

Os equipamentos utilizados na realização das soldagens foram (Figura 1):

- Robô industrial para deslocamento automático da tocha de soldagem (1).

- Uma fonte de soldagem eletrônica multiprocesso (2).

- Sistema de aquisição dos sinais de tensão e corrente operando em freqüência de 9600 Hz (3).

- Um medidor de velocidade de alimentação de arame (4).

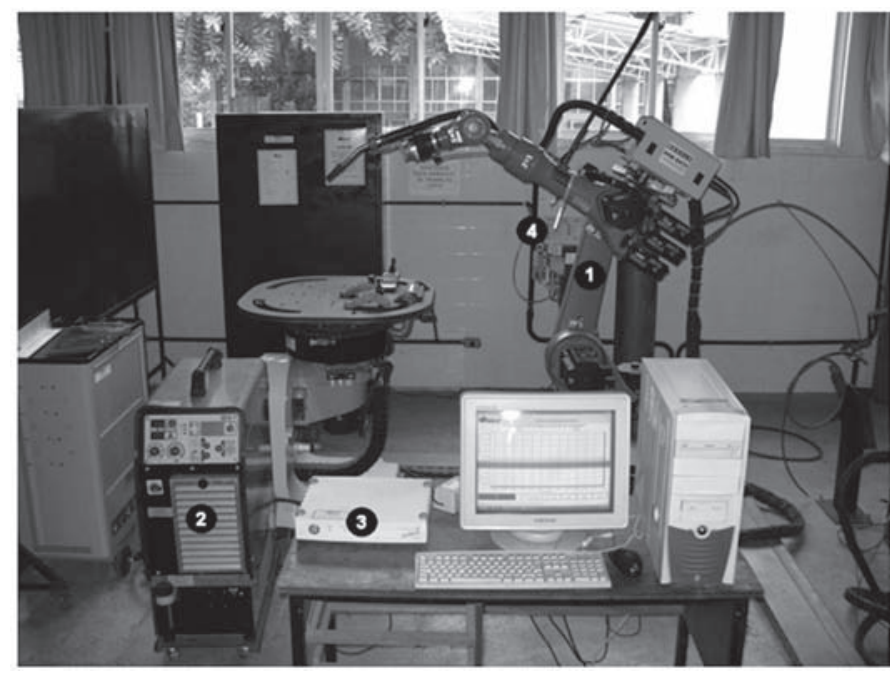

Figura 1 - Equipamentos utilizados nas soldagens: (1) robô industrial de soldagem, (2) fonte eletrônica multiprocesso, (3) Sistema de aquisição, (4) Medidor de Valim.

\subsection{Metodologia}

Durante o planejamento o número de ensaios a serem realizados, foi determinado pelo uso do método Taguchi. Assim Foram escolhidos seis fatores de controle com três níveis cada: Tensão de referência ( $\mathrm{Ur}=20,22,24 \mathrm{~V})$, Velocidade soldagem $(\mathrm{Vs}=0,2,0,3$ e 0,4 m/min), Tipo de tecimento (Sem tecimento ou ST, duplo oito ou D8 e Tecimento triangular ou TT mostrados na Figura 2), Técnica da tocha (normal, empurrando e puxando mostrados na Figura 3), Gás de proteção (100\%Ar, $70 \% \mathrm{Ar}+30 \% \mathrm{He}$ e $99,97 \% \mathrm{Ar}+0,03 \% \mathrm{NO})$ e o Material de adição (Liga= ERNiCrMo-3 ou 625, ERNiCrMo-14 ou 686 e ERNiCrMo-4 ou C-276). Tais fatores foram divididos pelo método Taguchi em duas matrizes L9, resultando num total de 18 ensaios [1].

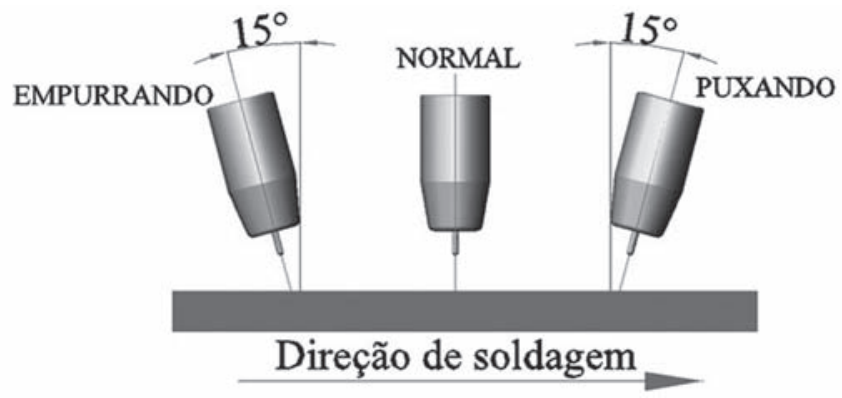

Figura 2 - Posições da tocha.

As soldagens foram realizadas na posição plana por simples deposição dos arames eletrodos sobre as placas de aço ASTM 516 Gr60. Em todos os ensaios foram mantidas constantes a vazão do gás de proteção em $20 \mathrm{~L} / \mathrm{min}$, a velocidade de alimentação em $6,0 \mathrm{~m} / \mathrm{min}$ e a DBCP (Distância Bico de Contato à Peça) em 20 mm. Nas Tabelas 1 e 2 encontram-se descritos: a seqüência de execução, os parâmetros de soldagem e os consumíveis empregados em cada ensaio das duas matrizes ortogonais L9 (propostas pelo método Taguchi). 


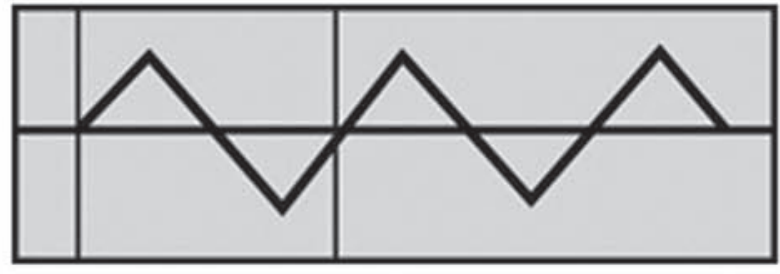

(a)

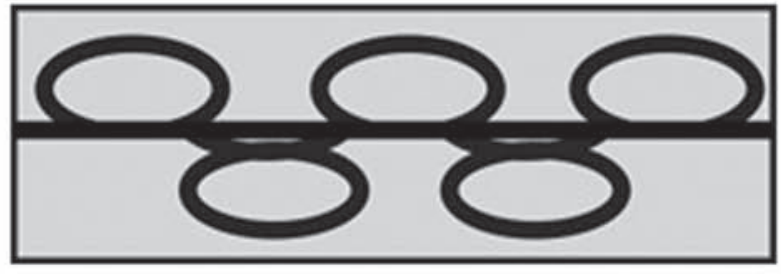

(b)

Figura 3 - Tipo de tecimento: (a)Tecimento triangular e (b) Tecimento em duplo oito.

Tabela 1. Ensaios realizados com a matriz L9-1.

\begin{tabular}{|c|c|c|c|c|}
\hline Ensaio & Ur $(\mathbf{V})$ & Técnica da Tocha & Tipo de tecimento & Vs (m/min) \\
\hline R01 & $1(20)$ & 1 (Normal) & 1 (Sem Tecimento) & $1(0,2)$ \\
\hline R02 & $1(20)$ & 2 (Empurrando) & 2 (Tec.Triangular) & $2(0,3)$ \\
\hline R03 & $1(20)$ & 3 (Puxando) & 3 (Duplo oito ) & $3(0,4)$ \\
\hline R04 & $2(22)$ & 1 (Normal) & 2 (Tec.Triangular) & $3(0,4)$ \\
\hline R05 & $2(22)$ & 2 (Empurrando) & 3 (Duplo oito ) & $1(0,2)$ \\
\hline R06 & $2(22)$ & 3 (Puxando) & 1 (Sem Tecimento) & $2(0,3)$ \\
\hline R07 & $3(24)$ & 1 (Normal) & 3 (Duplo oito ) & $2(0,3)$ \\
\hline R08 & $3(24)$ & 2 (Empurrando) & 1 (Sem Tecimento) & $3(0,4)$ \\
\hline R09 & $3(24)$ & 3 (Puxando) & 2(Tec.Triangular) & $1(0,2)$ \\
\hline
\end{tabular}

Tabela 2. Ensaios realizados com a matriz L9-2

\begin{tabular}{|c|c|c|c|c|}
\hline Ensaio & Liga & Gás de proteção & Vs (m/min) & Ur (V) \\
\hline R10 & $1(625)$ & $1(100 \% \mathrm{Ar})$ & $1(0,2)$ & $1(20)$ \\
\hline R11 & $1(625)$ & $2(70 \% \mathrm{Ar}+30 \% \mathrm{He})$ & $2(0,3)$ & $2(22)$ \\
\hline R12 & $1(625)$ & $3(99,97 \% \mathrm{Ar}+0,03 \% \mathrm{NO})$ & $3(0,4)$ & $3(24)$ \\
\hline R13 & $2(686)$ & $1(100 \% \mathrm{Ar})$ & $2(0,3)$ & $3(24)$ \\
\hline R14 & $2(686)$ & $2(70 \% \mathrm{Ar}+30 \% \mathrm{He})$ & $3(0,4)$ & $1(20)$ \\
\hline R15 & $2(686)$ & $3(99,97 \% \mathrm{Ar}+0,03 \% \mathrm{NO})$ & $1(0,2)$ & $2(22)$ \\
\hline R16 & $3(\mathrm{C}-276)$ & $1(100 \% \mathrm{Ar})$ & $3(0,4)$ & $2(22)$ \\
\hline R17 & $3(\mathrm{C}-276)$ & $2(70 \% \mathrm{Ar}+30 \% \mathrm{He})$ & $1(0,2)$ & $3(24)$ \\
\hline R18 & $3(\mathrm{C}-276)$ & $3(99,97 \% \mathrm{Ar}+0,03 \% \mathrm{NO})$ & $2(0,3)$ & $1(20)$ \\
\hline
\end{tabular}

Então, como forma de comparar os gastos despendidos em cada um dos ensaios foi utilizado o custo direto total da soldagem de um revestimento com $55 \mathrm{~mm}$ de largura e $190 \mathrm{~mm}$ de comprimento, utilizando uma sobreposição de 50\%. Para o cálculo deste custo, foram necessárias três variáveis: a taxa de deposição (TD), a taxa de fusão (TF) e a eficiência de deposição (ED), que foram determinadas com base nas diferenças de peso dos corpos de prova das matrizes L9-1 e L9-2 antes e após as soldagens, e nas velocidades de soldagem e de alimentação do arame-eletrodo. As diferenças de pesos foram medidas com uma balança eletrônica com resolução de $0,1 \mathrm{~g}$.

O reforço do cordão de solda também foi utilizado neste trabalho para selecionar os parâmetros a serem utilizados na deposição do revestimento, pois os revestimentos internos empregados em tubulações para o setor de petróleo e gás natural exigem um reforço líquido de $3 \mathrm{~mm}$, por isso deve-se obter um reforço bruto o mais próximo possível de $5 \mathrm{~mm}$ afim de permitir uma usinagem posterior que resulte neste valor estipulado para o reforço líquido [2]. Assim, caso seja necessário o uso de duas camadas para atender ao requisito mínimo de reforço, o custo torna-se relevante para a escolha dos melhores parâmetros de soldagem para a obtenção de revestimentos.

\section{Resultados e Discussão}

\subsection{Custos de soldagem}

A Tabela 3 apresenta os valores da taxa de fusão (TF), da taxa de deposição (TD) e da eficiência de deposição (ED) obtidos para cada ensaio, as quais foram calculadas através da 
equação 1, da equação 2 e da equação 3 respectivamente. Esta tabela mostra ainda os valores da massa do corpo de prova antes da soldagem (P1), a massa do corpo de prova após a soldagem $(\mathrm{P} 2)$ e a diferença entre as massas $(\Delta \mathrm{P})$.

- Taxa de fusão do arame (TF):

$T F=\frac{3,6 \cdot \rho_{a} \cdot l_{a}}{t_{s}}\left(\frac{K g}{\mathrm{~h}}\right)$

Onde:

$\rho_{\mathrm{a}}$ é a densidade linear do arame $(\mathrm{g} / \mathrm{m})$

$1_{a}$ é o comprimento do arame consumido (m)

$\mathrm{t}_{\mathrm{s}}$ é o tempo de soldagem (s)
- Taxa de deposição do arame (TD):

$T D=\frac{3,6 \cdot\left(m_{f}-m_{i}\right)}{t_{s}}\left(\frac{K g}{\mathrm{~h}}\right)$

Onde:

$\mathrm{m}_{\mathrm{f}}$ é a massa inicial da chapa ( $\mathrm{g}$ )

$\mathrm{m}_{\mathrm{i}}$ é a massa final da chapa $(\mathrm{g})$

$\mathrm{t}_{\mathrm{s}}$ é o tempo de soldagem (s)

- Eficiência de deposição (ED):

$E D=\frac{T D}{T F} \times 100(\%)$

Tabela 3. Valores obtidos para a TD, TF e ED

\begin{tabular}{|c|c|c|c|c|c|c|}
\hline Ensaio & $\mathbf{P 1}(\mathbf{g})$ & $\mathbf{P 2}(\mathbf{g})$ & $\Delta \mathbf{P}(\mathbf{g})$ & $\mathbf{T D}(\mathbf{k g} / \mathbf{h})$ & $\mathbf{T F} \mathbf{( k g} / \mathbf{h})$ & $\mathbf{E D ~ \%}$ \\
\hline R01 & 1044,4 & 1076,0 & 31,6 & 2,9 & 3,0 & 96,8 \\
\hline R02 & 1034,4 & 1055,1 & 20,7 & 2,9 & 3,0 & 94,8 \\
\hline R03 & 1039,9 & 1055,6 & 15,7 & 2,9 & 3,1 & 95,1 \\
\hline R04 & 1099,9 & 1115,7 & 15,8 & 2,9 & 3,1 & 95,7 \\
\hline R05 & 1022,9 & 1054,1 & 31,2 & 2,9 & 3,0 & 95,7 \\
\hline R06 & 1050,5 & 1072,5 & 20,8 & 2,9 & 3,0 & 95,2 \\
\hline R07 & 1047,5 & 1068,4 & 20,9 & 2,9 & 3,0 & 95,7 \\
\hline R08 & 1052,6 & 1068,6 & 16,0 & 3,0 & 3,1 & 96,9 \\
\hline R09 & 1024,4 & 1055,8 & 31,4 & 2,9 & 3,0 & 96,3 \\
\hline R10 & 1001,9 & 1032,8 & 30,9 & 2,9 & 3,0 & 94,8 \\
\hline R11 & 996,1 & 1016,6 & 20,5 & 2,8 & 3,0 & 93,9 \\
\hline R12 & 1004,3 & 1019,8 & 15,5 & 2,9 & 3,1 & 93,9 \\
\hline R13 & 976,0 & 997,5 & 21,5 & 3,0 & 3,0 & 98,0 \\
\hline R14 & 990,9 & 1007,0 & 16,1 & 3,0 & 3,1 & 97,5 \\
\hline R15 & 994,5 & 1026,7 & 32,2 & 3,0 & 3,0 & 99,1 \\
\hline R16 & 981,5 & 996,7 & 15,2 & 2,8 & 3,1 & 92,1 \\
\hline R17 & 1025,2 & 1055,8 & 30,6 & 2,8 & 3,0 & 93,9 \\
\hline R18 & 1037,2 & 1057,0 & 19,8 & 2,7 & 3,0 & 90,6 \\
\hline RC01 & 994,0 & 1025,1 & 31,1 & 2,9 & 3,0 & 95,4 \\
\hline RC02 & 963,8 & 994,4 & 30,6 & 2,8 & 3,0 & 93,9 \\
\hline RC03 & 1023,8 & 1054,6 & 30,8 & 2,8 & 3,0 & 94,8 \\
\hline RC04 & 959,0 & 990,8 & 31,8 & 2,9 & 3,1 & 95,6 \\
\hline RC05 & 968,9 & 999,8 & 30,9 & 2,9 & 3,1 & 93,6 \\
\hline
\end{tabular}

Com os resultados da Tabela 3 e os dados relacionados abaixo, os custos diretos da soldagem MIG/MAG, em R $\$ / \mathrm{kg}$ de solda, foram calculados de acordo com as equações 4 a 9 e encontram-se listados na Tabela 4 [3].

$\checkmark$ Custo unitário do gás $(\mathrm{G})$ :

- - Argônio puro: 31,58 R $\$ / \mathrm{m}^{3}$;

- - 70\% de argônio + 30\% de hélio: 45,00 R \$ $/ \mathrm{m}^{3}$;

- - 99,97\% de argônio + 0,03 \% de óxido nítrico: $35,00 \mathrm{R} \$ / \mathrm{m}^{3}$;
Vazão do gás $(\mathrm{F}): 1,5 \mathrm{~m}^{3} / \mathrm{h}$;

$\checkmark$ Custo da energia (P): 0,56 R $\$ / \mathrm{kWh}$;

$\checkmark$ Custo da arme-eletrodo (M):

- - AWS ER NiCrMo-3 (625): 217,90 R \$/kg;

- - AWS ER NiCrMo-4 (C-276): 317,31 R\$/kg;

- - AWS ER NiCrMo-14 (686): 564,43 R\$/kg;

Salário do soldador (S): $10 \mathrm{R} \$ / \mathrm{h}$;

$\checkmark$ Fator de operação (K): 50\%;

$\checkmark$ Custos gerais $(\mathrm{O}): 1 \mathrm{R} \$ / \mathrm{h}$. 
- Custo do gás $\left(\mathrm{C}_{\mathrm{G}}\right)$ por unidade de peso de metal depositado $(\mathrm{R} \$ / \mathrm{kg})$

$$
C_{G}=\frac{G \times F}{T D}
$$

Onde:

$\mathrm{TD}=$ taxa de deposição $(\mathrm{kg} / \mathrm{h})$;

$\mathrm{F}=$ vazão de gás $\left(\mathrm{m}^{3} / \mathrm{h}\right)$;

$\mathrm{G}=$ custo unitário de gás $\left(\mathrm{R} \$ / \mathrm{m}^{3}\right)$

- Custo da energia $\left(\mathrm{C}_{\mathrm{E}}\right)$ por unidade de peso de metal depositado $(\mathrm{R} \$ / \mathrm{kg})$

$$
C_{E}=\frac{P \times V \times A}{1000 \times T D}
$$

Onde:

$\mathrm{A}=$ corrente de soldagem $(\mathrm{A})$;

$\mathrm{V}=$ tensão $(\mathrm{V})$;

$\mathrm{P}=$ custo da energia $(\mathrm{R} \$ / \mathrm{kWh})$.

- Custo do arame-eletrodo $\left(\mathrm{C}_{\mathrm{AE}}\right)$ por unidade de peso de metal depositado $(\mathrm{R} \$ / \mathrm{kg})$

$$
\begin{aligned}
C_{A E}= & \frac{M}{E D} \\
& \text { Onde: } \\
& \mathrm{M}=\text { custo do arame-eletrodo }(\mathrm{R} \$ / \mathrm{kg}) ; \\
& \mathrm{ED}=\text { eficiência de deposição }(\%) ;
\end{aligned}
$$

- Custo do soldador $\left(\mathrm{C}_{\mathrm{s}}\right)$ por unidade de peso de metal depositado $(\mathrm{R} \$ / \mathrm{kg})$

$$
\begin{aligned}
C_{S}= & \frac{S \times K}{T D \times 100} \\
& \text { Onde: } \\
& \mathrm{K}=\text { fator de operação }(\%) ; \\
& \mathrm{S}=\text { Salário do soldador }(\mathrm{R} \$ / \mathrm{h}) ;
\end{aligned}
$$

\begin{tabular}{|c|c|c|c|c|c|c|}
\hline Ensaio & $\begin{array}{l}\text { Custo gás } \\
\text { (R\$/kg) }\end{array}$ & $\begin{array}{c}\text { Custo energia } \\
\text { (R\$/kg) }\end{array}$ & $\begin{array}{c}\text { Custo materiais } \\
(\mathrm{R} \$ / \mathbf{k g})\end{array}$ & $\begin{array}{c}\text { Custo Trabalho } \\
\text { (R\$/kg) }\end{array}$ & $\begin{array}{c}\text { Custos gerais } \\
\text { (R\$/kg) }\end{array}$ & $\begin{array}{l}\text { Custo direto total } \\
\text { (R\$/kg) }\end{array}$ \\
\hline R01 & 16,24 & 0,58 & 225,02 & 1,71 & 0,69 & 244,24 \\
\hline R02 & 16,53 & 0,55 & 229,94 & 1,74 & 0,70 & 249,46 \\
\hline R03 & 16,34 & 0,56 & 229,17 & 1,73 & 0,69 & 248,48 \\
\hline R04 & 16,24 & 0,58 & 227,72 & 1,71 & 0,69 & 246,94 \\
\hline R05 & 16,45 & 0,60 & 227,64 & 1,74 & 0,69 & 247,11 \\
\hline R06 & 16,45 & 0,57 & 228,84 & 1,74 & 0,69 & 248,29 \\
\hline R07 & 16,37 & 0,59 & 227,74 & 1,73 & 0,69 & 247,12 \\
\hline R08 & 16,04 & 0,55 & 224,87 & 1,69 & 0,68 & 243,83 \\
\hline R09 & 16,34 & 0,58 & 226,19 & 1,73 & 0,69 & 245,52 \\
\hline R10 & 16,61 & 0,60 & 229,85 & 1,75 & 0,70 & 249,50 \\
\hline R11 & 23,78 & 0,69 & 232,19 & 1,76 & 0,70 & 259,13 \\
\hline R12 & 18,35 & 0,63 & 232,13 & 1,75 & 0,70 & 253,55 \\
\hline $\mathrm{R} 13$ & 15,91 & 0,59 & 575,72 & 1,68 & 0,67 & 594,58 \\
\hline R14 & 22,71 & 0,61 & 578,87 & 1,68 & 0,67 & 604,55 \\
\hline R15 & 17,66 & 0,61 & 569,83 & 1,68 & 0,67 & 590,45 \\
\hline R16 & 16,88 & 0,57 & 344,70 & 1,78 & 0,71 & 364,65 \\
\hline R17 & 23,90 & 0,70 & 337,99 & 1,77 & 0,71 & 365,06 \\
\hline $\mathrm{R} 18$ & 19,15 & 0,56 & 350,07 & 1,82 & 0,73 & 372,33 \\
\hline $\mathrm{RC} 01$ & 31,35 & 0,59 & 228,37 & 1,74 & 0,70 & 262,75 \\
\hline $\mathrm{RC} 02$ & 31,86 & 0,65 & 232,10 & 1,77 & 0,71 & 267,09 \\
\hline $\mathrm{RC} 03$ & 16,66 & 0,56 & 334,91 & 1,76 & 0,70 & 354,59 \\
\hline $\mathrm{RC} 04$ & 23,00 & 0,62 & 228,05 & 1,70 & 0,68 & 254,06 \\
\hline $\mathrm{RC} 05$ & 16,61 & 0,53 & 339,12 & 1,75 & 0,70 & 358,72 \\
\hline
\end{tabular}

- Custos gerais $\left(\mathrm{C}_{\mathrm{Ge}}\right)$ por unidade de peso de metal depositado $(\mathrm{R} \$ / \mathrm{kg})$

$$
\begin{aligned}
C_{G e}= & \frac{O}{T D \times(K / 100)} \\
\text { Onde: } & \\
& \mathrm{O}=\text { custos gerais }(\mathrm{R} \$ / \mathrm{h}) ;
\end{aligned}
$$

- Custo direto total $\left(\mathrm{C}_{\mathrm{DT}}\right)$ por unidade de peso de metal depositado $(\mathrm{R} \$ / \mathrm{kg})$

$$
C_{D T}=\frac{C_{G}+C_{E}+C_{A E}+C_{S}+C_{G E}}{T D \times(K / 100)}
$$

Tabela 4. Custos diretos da soldagem para cada ensaio. 
Para a determinação do custo direto total da soldagem de revestimento, foi necessário conhecer o número de passes "n" e o tempo de soldagem contínua. O número de passes " $n$ " para um revestimento com largura total "LT", produzido por um cordão de solda de largura "L", (Figura 4), foi determinado pela equação 10 , enquanto que o tempo de soldagem contínua para a realização do revestimento foi determinado pela equação 11 .

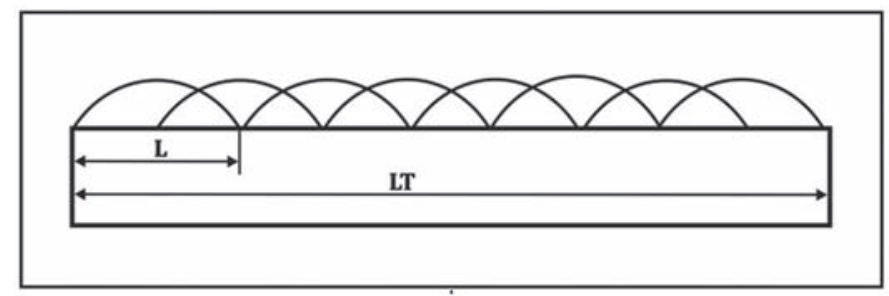

Figura 4 - Esquema de um revestimento com largura LT produzido por um cordão de solda de largura L, considerando uma sobreposição de 50\% (L/2).

$$
\begin{gathered}
\mathrm{n}=\frac{2 \times \mathrm{LT}-\mathrm{L}}{\mathrm{L}} \\
\mathrm{t}_{\mathrm{T}}=\left[\frac{\mathrm{C}}{\mathrm{Vs}} \times\left(\frac{2 \times \mathrm{LT}-\mathrm{L}}{\mathrm{L}}\right)\right]
\end{gathered}
$$

Onde:

$\mathrm{t}_{\mathrm{T}}=$ tempo total de soldagem contínua;

$\mathrm{C}=$ comprimento do cordão de solda;

$\mathrm{V}_{\mathrm{S}}=$ velocidade de soldagem.

Como o reforço bruto do revestimento deve ter no mínimo $5 \mathrm{~mm}$ de altura e considerando que o reforço máximo obtido nos cordões individuais dos ensaios para as duas matrizes ortogonais foi de 4,0 mm (Ensaio R3), conclui-se que os revestimentos devem ter no mínimo duas camadas. Contudo a altura final de um revestimento com mais de uma camada não é simplesmente a soma do reforço do cordão isolado, pois dois fenômenos contribuem para a sua altura final. O primeiro se refere à diferença de molhabilidade do metal de solda no substrato, pois, na primeira camada o substrato é o aço ASTM A 516 Gr 60, enquanto na segunda, é o próprio material do metal de solda que proporciona uma maior molhabilidade, resultando num cordão de solda mais largo e com um reforço menor. $\mathrm{O}$ outro fenômeno envolvido é relativo ao efeito da sobreposição ( $50 \%$ neste trabalho) dos cordões que proporciona um aumento da altura do revestimento à medida que esta aumenta. Assim a título de comparação, não foi considerado o efeito destes dois fenômenos. Desta forma, o custo direto total do revestimento (Custo), considerando a soldagem contínua para cada ensaio foi determinado pela multiplicação do custo direto total pelo tempo total de soldagem contínua $\left(\mathrm{t}_{\mathrm{T}}\right)$ e pela taxa de deposição (TD). A Tabela 5 apresenta os valores previstos de algumas variáveis (diluição, reforço, número de passes, o tempo total de soldagem contínua e custo direto total do revestimento) em cada ensaio para a primeira camada (variáveis com índice subscrito 1) e para a segunda camada (variáveis com índice subscrito 2). Tendo em vista que um revestimento com uma terceira camada seria muito oneroso, não foi feita uma previsão das variáveis para esta condição.

Com base nos resultados mostrados na Tabela 5, foi montada a Figura 5 que nos mostra graficamente os custos $\left(\mathrm{Custo}_{2}\right)$, os reforços $\left(\mathrm{R}_{2}\right)$ e as diluições $\left(\mathrm{D}_{2}\right)$ previstos para duas camadas em função do ensaio e, nesta figura, foi traçada uma linha de referência em um reforço de $5 \mathrm{~mm}$, para escolha dos ensaios que satisfazem ou se aproximam desta condição. Assim, conforme esta figura, apenas os ensaios R7, R11, R12 e RC4 proporcionam reforços próximos ao valor de referência e com custos aceitáveis.

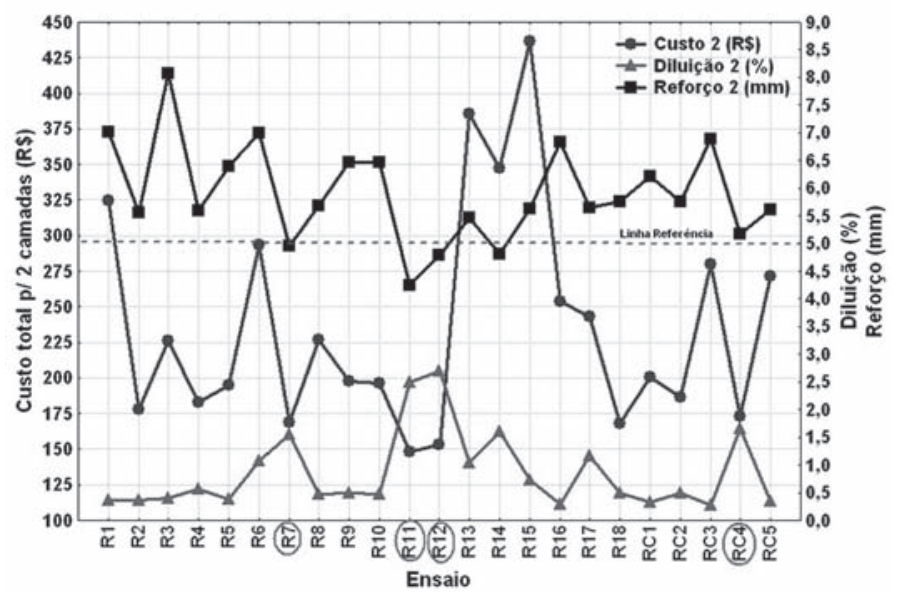

Figura 5 - Custo total, reforço e diluição previstos para duas camadas em função dos ensaios.

Em todos os quatro ensaios pré-selecionados (R7, R11, R12 e RC4), o material de adição utilizado, foi a liga 625 , que apresenta um custo do arame-eletrodo muito inferior às demais ligas (C-276 e 686). Assim a escolha do material de adição para aplicação do revestimento ficou restrita somente à liga 625 , pois os custos foram calculados com base apenas nos custos relativos à soldagem sem levar em consideração os custos relativos à corrosão. Contudo vale ressaltar que as operações de revestimento na indústria de petróleo e gás natural são utilizadas de forma geral para melhorar resistência à corrosão dos equipamentos e componentes. Desse modo, uma escolha mais rigorosa da liga deve incluir também ensaios de corrosão para comparar os custos relacionados ao desgaste por corrosão destas ligas na vida útil dos revestimentos. Os cálculos dos custos relacionados à corrosão ficam como uma sugestão para trabalhos futuros.

A Tabela 6 apresenta de forma mais detalhada os valores previstos para o custo, o reforço e a diluição dos ensaios préselecionados para execução das soldagens relativas aos ensaios de revestimentos. 
Tabela 5. Valores previstos para: a diluição (D), o reforço (R), o número de passes (n), o tempo total de soldagem contínua (tT) e o custo direto total do revestimento (Custo).

\begin{tabular}{|c|c|c|c|c|c|c|c|c|c|c|}
\hline \multirow[b]{2}{*}{ Ensaio } & \multicolumn{5}{|c|}{$1^{a}$ camada } & \multicolumn{5}{|c|}{$2^{\mathrm{a}}$ camada } \\
\hline & $D_{1}(\%)$ & $\begin{array}{c}R_{1} \\
(\mathbf{m m})\end{array}$ & $n_{1}$ & $\begin{array}{c}\mathbf{t}_{\mathrm{T} 1} \\
(\mathrm{~min})\end{array}$ & $\begin{array}{c}\text { Custo }_{1} \\
\text { (R\$) }\end{array}$ & $\begin{array}{l}D_{2} \\
(\%)\end{array}$ & $\begin{array}{c}\mathbf{R}_{2} \\
(\mathbf{m m})\end{array}$ & $\begin{array}{c}\mathbf{t}_{\mathrm{T} 2} \\
(\mathrm{~min})\end{array}$ & $\mathbf{n}_{2}$ & $\begin{array}{c}\text { Custo }_{2} \\
\text { (R\$) }\end{array}$ \\
\hline R01 & 6,1 & 3,5 & 21 & 13,7 & 162,28 & 0,4 & 7,0 & 27,3 & 42 & 324,56 \\
\hline R02 & 6,0 & 2,8 & 17 & 7,5 & 89,15 & 0,4 & 5,6 & 15,0 & 34 & 178,31 \\
\hline R03 & 6,4 & 4,0 & 29 & 9,4 & 113,08 & 0,4 & 8,1 & 18,8 & 58 & 226,15 \\
\hline R04 & 7,6 & 2,8 & 23 & 7,6 & 91,38 & 0,6 & 5,6 & 15,2 & 46 & 182,75 \\
\hline R05 & 6,2 & 3,2 & 13 & 8,2 & 97,47 & 0,4 & 6,4 & 16,4 & 26 & 194,94 \\
\hline R06 & 10,4 & 3,5 & 28 & 12,3 & 146,62 & 1,1 & 7,0 & 24,6 & 56 & 293,23 \\
\hline R07 & 12,5 & 2,5 & 16 & 7,1 & 84,23 & 1,6 & 5,0 & 14,1 & 32 & 168,46 \\
\hline R08 & 7,0 & 2,9 & 29 & 9,4 & 113,34 & 0,5 & 5,7 & 18,9 & 58 & 226,69 \\
\hline R09 & 7,0 & 3,2 & 13 & 8,3 & 98,77 & 0,5 & 6,5 & 16,7 & 26 & 197,55 \\
\hline R10 & 6,8 & 3,2 & 13 & 8,3 & 98,34 & 0,5 & 6,5 & 16,6 & 26 & 196,68 \\
\hline R11 & 15,8 & 2,1 & 14 & 6,0 & 74,00 & 2,5 & 4,2 & 12,1 & 28 & 148,00 \\
\hline R12 & 16,5 & 2,4 & 20 & 6,4 & 76,77 & 2,7 & 4,8 & 12,7 & 40 & 153,54 \\
\hline $\mathrm{R} 13$ & 10,2 & 2,7 & 15 & 6,5 & 192,65 & 1,0 & 5,5 & 13,1 & 30 & 385,31 \\
\hline R14 & 12,7 & 2,4 & 18 & 5,8 & 173,57 & 1,6 & 4,8 & 11,6 & 36 & 347,13 \\
\hline R15 & 8,6 & 2,8 & 11 & 7,5 & 218,35 & 0,7 & 5,6 & 14,9 & 22 & 436,70 \\
\hline R16 & 5,4 & 3,4 & 23 & 7,4 & 126,74 & 0,3 & 6,8 & 14,9 & 46 & 253,48 \\
\hline R17 & 10,8 & 2,8 & 11 & 7,1 & 121,58 & 1,2 & 5,7 & 14,2 & 22 & 243,16 \\
\hline R18 & 7,9 & 2,9 & 11 & 5,0 & 84,61 & 0,6 & 5,8 & 10,0 & 22 & 169,21 \\
\hline RC01 & 5,7 & 3,1 & 12 & 8,0 & 100,38 & 0,3 & 6,2 & 16,0 & 24 & 200,77 \\
\hline $\mathrm{RC} 02$ & 7,0 & 2,9 & 11 & 7,4 & 93,16 & 0,5 & 5,8 & 14,8 & 22 & 186,33 \\
\hline $\mathrm{RC} 03$ & 5,2 & 3,5 & 13 & 8,3 & 140,03 & 0,3 & 6,9 & 16,7 & 26 & 280,06 \\
\hline $\mathrm{RC} 04$ & 12,9 & 2,6 & 11 & 7,0 & 86,58 & 1,7 & 5,2 & 13,9 & 22 & 173,16 \\
\hline $\mathrm{RC} 05$ & 5,8 & 2,8 & 12 & 8,0 & 135,83 & 0,3 & 5,6 & 15,9 & 24 & 271,65 \\
\hline
\end{tabular}

Tabela 6. Valores do reforço, do custo e da diluição para os ensaios pré-selecionados para o revestimento

\begin{tabular}{|c|c|c|c|}
\hline Ensaio & $\mathbf{R}_{\mathbf{2}}(\mathbf{m m})$ & $\mathbf{D}_{\mathbf{2}}(\mathbf{\%})$ & Custo $_{\mathbf{2}}$ (R\$) \\
\hline $\mathrm{R} 07$ & 5,0 & 1,6 & 168,46 \\
\hline $\mathrm{R} 11$ & 4,2 & 2,5 & 148,00 \\
\hline $\mathrm{R} 12$ & 4,8 & 2,7 & 153,54 \\
\hline $\mathrm{RC} 04$ & 5,2 & 1,7 & 173,16 \\
\hline
\end{tabular}

Ao analisar-se a Tabela 6, verifica-se que os quatro ensaios pré-selecionados obtiveram baixos valores de diluição, inferiores a $3 \%$ e valores de reforço próximos a $5 \mathrm{~mm}$, o que os torna aptos à soldagem dos revestimentos. Então para decidir o ensaio adequado, foi utilizado o critério do menor custo, o que leva a escolha do Ensaio R11.

\section{Conclusões}

Com base nos resultados experimentais apresentados neste trabalho foi possível concluir que:

- Todas as combinações dos fatores de controle conduziriam a soldagens de revestimento com uma camada, as quais obteriam um reforço dos cordões de solda inferiores a $4 \mathrm{~mm}$, sendo necessária a aplicação de uma segunda para atender à exigência de $5 \mathrm{~mm}$ de reforço bruto da norma N-1707 (PETROBRAS);

- O uso do custo de soldagem demonstrou ser um parâmetro útil no auxílio da avaliação e seleção dos parâmetros de soldagem a serem utilizados nas operações de revestimento;

- A escolha do material de adição para aplicação do revestimento ficou restrita somente à liga 625 , que apresenta um custo do arame-eletrodo muito inferior às demais ligas (C-276 e 686);

- Os parâmetros de soldagem utilizados no ensaio R11 (Liga $=625$, Gás $=70 \% \mathrm{Ar}+30 \% \mathrm{He}, \mathrm{Vs}=0,3 \mathrm{~m} / \mathrm{min}$ e Ur $=22$ $\mathrm{V})$ obtiveram o menor custo para o revestimento com duas 
camadas ( $\mathrm{R} \$ 148,00)$ e um reforço compatível com o mínimo exigido na norma $\mathrm{N}-1707$.

\section{Agradecimentos}

Os autores gostariam de agradecer ao Laboratório de Engenharia de Soldagem, ao Laboratório de Caracterização de Materiais pertencentes à Universidade Federal do Ceará pelo suporte na realização dos experimentos, ao PRH-31(ANP) e ao CENPES (Petrobras) pelo suporte financeiro.

\section{Referências Bibliográficas}

[1] ROSS, P. J. Aplicações das Técnicas Taguchi na Engenharia da Qualidade São Paulo - SP: McGraw-Hill Ltda. 1991. 333 p.

[2] PETROBRAS N -1707. Projeto de vaso de pressão com revestimento. Rio de Janeiro - RJ: PETRÓLEO BRASILEIRO S.A. 1999.

[3] AWS. Welding Handbook - Welding Processes 9.ed. Miami: American Welding Society, v.2. 2004. 